\title{
MODULES OVER THE RING OF PSEUDORATIONAL NUMBERS AND QUOTIENT DIVISIBLE GROUPS
}

\author{
A. V. TSAREV
}

\begin{abstract}
Structure theorems are obtained for some classes of modules over the ring of pseudorational numbers and some classes of quotient divisible mixed groups.
\end{abstract}

\section{INTRODUCTION}

The ring of pseudorational numbers and modules over this ring were introduced by Fomin in 2 and Krylov in 12, 13. Later, in 3, 4 Fomin applied such modules to the study of quotient divisible mixed groups and torsion free groups of finite rank. In [12], Krylov used modules over the ring of pseudorational numbers for the study of the so-called $s p$-groups.

Many important Abelian groups are additive groups of modules over the ring of pseudorational numbers. The periodic, divisible, and algebraically compact groups and the groups with $\pi$-regular endomorphism ring are classical examples (all these classes were considered in [1]). The mixed groups contained in the direct product of their $p$ components (called the $s p$-groups) give another important example. The $s p$-groups were studied in the papers [5]-12]; this list is far from being exhaustive.

Besides 2], modules over the ring of pseudorational numbers were treated in 14, where the injective modules were described, and in [13, where the ideals of the ring of pseudorational numbers were described and some questions about endomorphism rings and groups of homomorphisms for $s p$-groups were answered.

This paper can be divided into three parts. In the first part $(\S \S 1,2)$ we introduce the principal notions and properties of modules over the ring of pseudorational numbers. $\S \S 3$ and 4 constitute the second part. In these sections we study the structure of some classes of finitely generated modules. Theorems 3.1 and 4.1 and Corollaries 3.1 and 4.1 are the main results of that part. In the concluding part $(\S 5)$ we discuss relationships between modules over the ring of pseudorational numbers and quotient divisible mixed groups and torsion free groups of finite rank.

Throughout the paper, $Z, Q$, and $\widehat{Z}_{p}$ denote (respectively) the rings of integers, rational numbers, and $p$-adic integers, as well as the additive groups of these rings. Next, $Z(m)$ stands for the residue class ring modulo $m, P$ denotes the set of all prime integers, and $\mathbb{N}$ is the set of positive integers. By $r(G)$ we denote the torsion free rank of the group $G$, which will be called simply the rank of $G$, and $r^{*}(M)$ is the pseudorational rank of an $R$-module $M$. The periodic part of a group $G$ will be denoted by $t(G)$. For a subset $S$ of a group $G$, we denote by $\langle S\rangle_{*}$ the pure hull of $S$ in $G$, i.e., the set of all $g \in G$ such that $m g \in\langle S\rangle$ for some nonzero integer $m$. The remaining notation and definitions are standard and correspond to those in [1].

2000 Mathematics Subject Classification. Primary 16L99.

Key words and phrases. Abelian groups, quotient divisible groups, ring of pseudorational numbers, pseudorational rank, module of pseudorational relations. 


\section{$\S 1$. ThE RING OF PSEUDORATIONAL NUMBERS}

In the ring $\widehat{Z}=\prod_{p \in P} \widehat{Z}_{p}$, we consider the subring generated as a pure subgroup by the ideal $\bigoplus_{p \in P} \widehat{Z}_{p}$ and the unity of the ring.

Definition 1.1. The ring $R=\left\langle 1, \bigoplus_{p \in P} \widehat{Z}_{p}\right\rangle_{*}$ is called the ring of pseudorational numbers.

We shall also use some other rings introduced in the papers [2] and [13]. Let $\chi=\left(m_{p}\right)$ be an arbitrary characteristic, and let $K_{p}=Z / p^{m_{p}} Z$ if $m_{p}<\infty$ and $K_{p}=\widehat{Z}_{p}$ otherwise. If the characteristic $\chi$ contains infinitely many nonzero components, then we denote by $R_{\chi} \subset \prod_{p \in P} K_{p}$ the subring whose additive group is the pure hull $\left\langle 1, \bigoplus_{p \in P} K_{p}\right\rangle_{*}$. If the $p$-components of $\chi$ are nonzero only for $p=p_{1}, \ldots, p_{n}$, then we put $K_{\chi}=K_{p_{1}} \oplus \cdots \oplus K_{p_{n}}$ and $R_{\chi}=Q \oplus K_{\chi}$. Observe that if $\chi=(\infty)$, then the ring $R_{\chi}$ is precisely the ring of pseudorational numbers.

The following properties of the rings $R_{\chi}$ were obtained in $[2$.

Properties:

$1^{0}$. An element $r=\left(\alpha_{p}\right) \in \prod_{p \in P} \widehat{Z}_{p}$ is contained in the ring $R$ if and only if there exists a rational number $|r|=m / n$ such that $n \alpha_{p}=m$ for almost all prime integers $p$.

$2^{0}$. For every $r \in R$ the rational number $|r|$ described in $1^{0}$ is unique.

$3^{0}$. The elements of the form $\varepsilon_{p}=\left(0, \ldots, 0,1_{p}, 0, \ldots\right)$ are idempotents of the ring R. Moreover, each idempotent of the ring of pseudorational numbers has the form $\varepsilon=$ $\varepsilon_{p_{1}}+\cdots+\varepsilon_{p_{n}}$ or $1-\varepsilon$.

$4^{0} . T=\bigoplus_{p \in P} \widehat{Z}_{p}$ is a maximal ideal of the ring of pseudorational numbers; it consists of all $r \in R$ such that $|r|=0$, and $R / T \cong Q$.

$5^{0}$. Each epimorphic image of the ring $R_{\chi}$ has the form $R_{\varphi}$ or $K_{\varphi}$, where $\chi \geq \varphi$.

Next we consider some invariants of modules over the ring of pseudorational numbers and recall some properties of such modules.

Definition $1.2([2])$. We say that an $R$-module $M$ is divisible if its additive group is torsion free and divisible and $r m=|r| m$ for all $r \in R, m \in M$. We say that an $R$-module is reduced if it contains no divisible submodules.

Theorem $1.1([2])$. Let $M$ be an arbitrary $R$-module. Then:

1. Either the module $M$ is reduced, or it contains the greatest divisible submodule $\operatorname{div} M$;

2. $\operatorname{div} M=\{m \in M \mid t m=0$ for every $t \in T\}$;

3. $\operatorname{div} M$ is a direct summand of $M$.

Theorem 1.2 ([2]). For an arbitrary $R$-module $M$, the set

$$
T M=\{t m \mid t \in T, m \in M\}
$$

is a submodule of the module $M$, and $T M=\bigoplus_{p \in P} M_{p}$, where $M_{p}=\varepsilon_{p} M$.

Let $M$ be a finitely generated $R$-module, and let $\left\{x_{1}, \ldots, x_{n}\right\}$ be a generating set of $M$. Obviously, the $\widehat{Z}_{p}$-module $M_{p}=\varepsilon_{p} M$ is generated by the elements $\left\{\varepsilon_{p} x_{1}, \ldots, \varepsilon_{p} x_{n}\right\}$. As any finitely generated $p$-adic module, $M_{p}$ decomposes into the direct sum of cyclic $\widehat{Z}_{p}$-modules:

$$
M_{p}=\left\langle a_{1}\right\rangle_{\widehat{Z}_{p}} \oplus \cdots \oplus\left\langle a_{n}\right\rangle_{\widehat{Z}_{p}} ;
$$

some of these modules may be zero. 
Any cyclic $\widehat{Z}_{p}$-module is isomorphic either to $\widehat{Z}_{p}$ or to $Z / p^{k_{i p}} Z$, where $k_{i p}$ is a nonnegative integer. Therefore, the isomorphism

$$
M_{p} \cong Z\left(p^{k_{1 p}}\right) \oplus \cdots \oplus Z\left(p^{k_{t p}}\right) \oplus \bigoplus_{s} \widehat{Z}_{p} \quad(t+s=n)
$$

gives rise to an ordered sequence of nonnegative integers and $\infty$ symbols,

$$
0 \leq k_{1 p} \leq \cdots \leq k_{n p} \leq \infty,
$$

the last $s$ terms of which are the $\infty$ symbols $(0 \leq s \leq n)$. The sequences (1.1) constructed for all prime integers $p$ determine a sequence of characteristics. Several terms at the beginning of this sequence may be the zero characteristics; deleting them, we obtain a sequence

$$
\chi_{1} \leq \cdots \leq \chi_{k} .
$$

The sequence of characteristics (1.2) will be called the generalized characteristic of the finitely generated $R$-module $M$.

Definition 1.3 ([2]). Let $M$ be an $R$-module; by the pseudorational rank of $M$ we mean the dimension $\operatorname{dim}_{Q}(M / T M)$ of the factor module $M / T M$ over the field $Q \cong R / T$.

Properties:

$6^{0}$. If $M=\left\langle x_{1}, \ldots, x_{n}\right\rangle_{R}$, then $r^{*}(M) \leq n$.

$7^{0}$. If $N$ is a submodule of an $R$-module $M$, then $r^{*}(N) \leq r^{*}(M)$.

Since $N \subseteq M$ and $T N \subseteq T M$, there exists a monomorphism of vector spaces $N / T N \rightarrow$ $M / T M$, which means that $\operatorname{dim}_{Q}(N / T M) \leq \operatorname{dim}_{Q}(M / T M)$.

$8^{0}$. If $N$ is a submodule of an $R$-module $M$, then $r^{*}(M)=r^{*}(M / N)+r^{*}(N)$.

Since $T(M / N)=T M / T N$ and $(M / N) /(T M / T N) \cong(M / T M) /(N / T N)$, we see that $r^{*}(M)=\operatorname{dim}_{Q}((M / N) /(T M / T N))+\operatorname{dim}_{Q}(N / T N)=r^{*}(M / N)+r^{*}(N)$.

$9^{0}$. If $M$ is an $R$-module whose generalized characteristic is locally free (i.e., it does not contain any $\infty$ symbol), then $r^{*}(M)=r(M)$.

If the generalized characteristic of the $R$-module $M$ is locally free, then $T M=t(M)$; hence, $r^{*}(M)=r(M / t(M))=r(M)$.

\section{§2. The module of PSEUdorational Relations}

Let $X=\left\{x_{1}, \ldots, x_{n}\right\}$ be any finite system of elements of an $R$-module $M$. Obviously, the set

$$
\Delta M_{X}=\left\{\left(r_{1}, \ldots, r_{n}\right) \in R^{n} \mid r_{1} x_{1}+\cdots+r_{n} x_{n}=0\right\}
$$

is an $R$-module. If $X$ is a generating system of $M$, then $\Delta M_{X}$ will be called the module of pseudorational relations of $M$.

Proposition 2.1. Let $M$ and $L$ be arbitrary modules over the ring $R$, let $X=\left\{x_{1}, \ldots, x_{n}\right\}$ be a generating system of $M$, and let $Y=\left\{y_{1}, \ldots, y_{n}\right\}$ be any system of elements of $L$. A homomorphism $f: M \rightarrow L$ such that $f\left(x_{i}\right)=y_{i}(1 \leq i \leq n)$ exists if and only if $\Delta M_{X} \subseteq \Delta L_{Y}$.

Proof. We prove the "only if" part. Let $f: M \rightarrow L$ be a homomorphism such that $f\left(x_{i}\right)=y_{i}(1 \leq i \leq n)$. If $\left(r_{1}, \ldots, r_{n}\right) \in \Delta M_{X}$, then $r_{1} x_{1}+\cdots+r_{n} x_{n}=0$, whence

$$
f\left(r_{1} x_{1}+\cdots+r_{n} x_{n}\right)=r_{1} y_{1}+\cdots+r_{n} y_{n}=0 .
$$

Thus, $\Delta M_{X} \subseteq \Delta L_{Y}$.

Now we prove the "if" part. Let $\Delta M_{X} \subseteq \Delta L_{Y}$; we construct a homomorphism $f: M \rightarrow L$ such that $f\left(x_{i}\right)=y_{i}(1 \leq i \leq n)$. 
We define a correspondence $f: M \rightarrow L$ by the rule

$$
f\left(r_{1} x_{1}+\cdots+r_{n} x_{n}\right)=r_{1} y_{1}+\cdots+r_{n} y_{n} .
$$

Let $g=r_{1} x_{1}+\cdots+r_{n} x_{n}=s_{1} x_{1}+\cdots+s_{n} x_{n}$ be two arbitrary representations of an element $g \in M$. Then

$$
\left(r_{1}-s_{1}\right) x_{1}+\cdots+\left(r_{n}-s_{n}\right) x_{n}=0,
$$

i.e., $\left(\left(r_{1}-s_{1}\right), \ldots,\left(r_{n}-s_{n}\right)\right) \in \Delta M_{X}$. The condition $\Delta M_{X} \subseteq \Delta L_{Y}$ implies

$$
\left(r_{1}-s_{1}\right) y_{1}+\cdots+\left(r_{n}-s_{n}\right) y_{n}=0
$$

which means that

$$
f\left(r_{1} x_{1}+\cdots+r_{n} x_{n}\right)=r_{1} y_{1}+\cdots+r_{n} y_{n}=s_{1} y_{1}+\cdots+s_{n} y_{n}=f\left(s_{1} x_{1}+\cdots+s_{n} x_{n}\right) .
$$

Thus, the correspondence $f$ is a mapping. Obviously, $f$ is compatible with the operations; i.e., $f$ is a homomorphism of $R$-modules. It remains to observe that $f\left(x_{i}\right)=y_{i}(1 \leq i \leq$ $n)$.

Corollary 2.1. Let $M$ and $L$ be finitely generated $R$-modules. They are isomorphic if and only if they admit equal modules of pseudorational relations 1

Proposition 2.2. Let $X=\left\{x_{1}, \ldots, x_{n}\right\}$ be a generating system of an $R$-module $M$; then

$$
n=r^{*}(M)+r^{*}\left(\Delta M_{X}\right) .
$$

Proof. Let $\varphi: R^{n} \rightarrow M$ be the mapping defined by the rule

$$
\varphi\left(r_{1}, \ldots, r_{n}\right)=r_{1} x_{1}+\cdots+r_{n} x_{n} .
$$

It is easily seen that $\varphi$ is a homomorphism. But $X$ is a generating system of the $R$-module $M$; hence, $\varphi$ is an epimorphism. Observe that

$$
\left(r_{1}, \ldots, r_{n}\right) \in \operatorname{ker} \varphi \Longleftrightarrow r_{1} x_{1}+\cdots+r_{n} x_{n}=0,
$$

i.e., $\operatorname{ker} \varphi=\Delta M_{X}$. Therefore,

$$
M \cong R^{n} / \Delta M_{X} \text { and } n=r^{*}(M)+r^{*}\left(\Delta M_{X}\right) .
$$

\section{§3. Modules of PSEUdorational Rank 1}

Lemma 3.1. If $M$ is a cyclic R-module, then its pseudorational rank is equal to 1 or 0 . In the first case $M \cong R_{\chi}$, where $\chi$ is an arbitrary characteristic, and in the second case $M \cong K_{\varphi}$, where $\varphi$ is an almost zero characteristic.

Proof. Let $M=\langle x\rangle_{R}$; then, obviously, the mapping $\varphi: R \rightarrow M$ defined by the rule $\varphi(r)=r x$ is an epimorphism of modules, and $\operatorname{ker} \varphi$ is an ideal of the ring $R$. Thus, the module $M$ is isomorphic to an epimorphic image of the ring $R$, which is isomorphic to one of the modules $R_{\chi}$ or $K_{\varphi}$.

Since $r^{*}\left(R_{\chi}\right)=1$ and $r^{*}\left(K_{\varphi}\right)=0$, we obtain the second statement of the lemma.

Lemma 3.2. If $M=\left\langle m_{1}, \ldots, m_{n}\right\rangle_{R}$ is a finitely generated $R$-module of pseudorational rank 1 , then we can choose a generating system $\left\{s_{1}, t_{2}, \ldots, t_{n}\right\}$ of the module $M$ so that $s_{1} \notin T M$ and $t_{2}, \ldots, t_{n} \in T M$.

\footnotetext{
${ }^{1}$ Translator's Note: Probably, here (as well as in the formulation of Proposition 5.1) the author has in mind the following: there exist generating systems of these modules for which the modules of their pseudorational relations coincide.
} 
Proof. We introduce the notation

$$
\begin{aligned}
m_{i_{1}} & =s_{1} \notin T M, \ldots, m_{i_{k}}=s_{k} \notin T M ; \\
m_{i_{k+1}} & =t_{k+1} \in T M, \ldots, m_{i_{n}}=t_{n} \in T M .
\end{aligned}
$$

Since

$$
\begin{aligned}
M / T M & =\left\langle s_{1}+T M, \ldots, s_{k}+T M, t_{k+1}+T M, \ldots, t_{n}+T M\right\rangle_{Q} \\
& =\left\langle s_{1}+T M, \ldots, s_{k}+T M\right\rangle_{Q} \text { and } \quad \operatorname{dim}_{Q} M / T M=1,
\end{aligned}
$$

we have $k \geq 1$. Since the module $M / T M$ is a one-dimensional vector space over $Q$, any two elements of $M$ are linearly dependent modulo $T M$ : if $l, m \notin T M$, then $l=r m+t$ for some $r \in R, t \in T M$. Hence, we can represent the elements $s_{1}, \ldots, s_{k}$ in the following form:

$$
\begin{aligned}
s_{1} & =s_{1} ; \\
s_{2} & =r_{2} s_{1}+t_{2}, \quad \text { where } r_{2} \in R, t_{2} \in T M ; \\
& \vdots \\
s_{k} & =r_{k} s_{1}+t_{k}, \quad \text { where } r_{k} \in R, t_{k} \in T M .
\end{aligned}
$$

It follows that $M=\left\langle s_{1}, \ldots, s_{k}, t_{k+1}, \ldots, t_{n}\right\rangle_{R}=\left\langle s_{1}, t_{2}, \ldots, t_{n}\right\rangle_{R}$.

Theorem 3.1. If $M$ is a finitely generated $R$-module of pseudorational rank 1 , then

$$
M \cong R_{\chi_{1}} \oplus K_{\chi_{2}} \oplus \cdots \oplus K_{\chi_{m}}
$$

where $\chi_{2}, \ldots, \chi_{m}$ are almost zero characteristics and $\chi_{1}$ is an arbitrary characteristic.

Proof. Let $M$ be an arbitrary $R$-module of pseudorational rank 1. By Lemma 3.2, we have $M=\left\langle s_{1}, t_{2}, \ldots, t_{n}\right\rangle_{R}$, where $s_{1} \notin T M$ and $t_{2}, \ldots, t_{n} \in T M$. Then

$$
M=\left\langle s_{1}, t_{2}, \ldots, t_{n}\right\rangle_{R}=\left\langle s_{1}\right\rangle_{R}+\left\langle t_{2}, \ldots, t_{n}\right\rangle_{R}=R s_{1}+S,
$$

where $S=\left\langle t_{2}, \ldots, t_{n}\right\rangle_{R} \subseteq T M$. Moreover, since $t_{2}, \ldots, t_{n} \in T M$, we can find an idempotent $\varepsilon \in R$ such that $S=\left\langle t_{2}, \ldots, t_{n}\right\rangle_{R} \subseteq \varepsilon M$. Then

$$
M=\left((1-\varepsilon) R s_{1}+\varepsilon R s_{1}\right)+S=(1-\varepsilon) R s_{1}+\left(\varepsilon R s_{1}+S\right)=(1-\varepsilon) R s_{1}+S_{1},
$$

where $S_{1}=\varepsilon R s_{1}+S$. Since $\varepsilon R s_{1}$ and $S$ are contained in $\varepsilon M$, the module $S_{1}$ is also contained in $\varepsilon M$, and $(1-\varepsilon) R s_{1} \cap S_{1}=0$ because $(1-\varepsilon) R s_{1} \cap \varepsilon M=0$. Therefore,

$$
M=(1-\varepsilon) R s_{1} \oplus S_{1} .
$$

Being a cyclic $R$-module of pseudorational rank 1 , the module $R s_{1}$ is isomorphic to a module of the form $R_{\chi}$. Then $(1-\varepsilon) R s_{1} \cong(1-\varepsilon) R_{\chi}=R_{\chi_{1}}$.

Let $\varepsilon=\varepsilon_{p_{1}}+\cdots+\varepsilon_{p_{t}}$. For all $i \in\{1, \ldots, t\}$, we define mappings $\pi_{i}: S_{1} \rightarrow \varepsilon_{p_{i}} S_{1}$ by the rules $\pi_{i}(s)=\varepsilon_{p_{i}} s$. These mappings satisfy the following conditions:

a) $\pi_{i} \pi_{j}= \begin{cases}0 & \text { if } i \neq j \\ \pi_{i} & \text { if } i=j\end{cases}$

b) for each $s \in S_{1}, s=\varepsilon s=\varepsilon_{p_{1}} s+\cdots+\varepsilon_{p_{t}} s=\pi_{p_{1}}(s)+\cdots+\pi_{p_{t}}(s)$.

It follows that $S_{1}=\pi_{p_{1}}\left(S_{1}\right) \oplus \cdots \oplus \pi_{p_{t}}\left(S_{1}\right)=\varepsilon_{p_{1}} S_{1} \oplus \cdots \oplus \varepsilon_{p_{t}} S_{1}$.

Since $\varepsilon_{p_{i}} R_{\chi_{1}}=0$ for each $i \in\{1, \ldots, t\}$, we conclude that

$$
\varepsilon_{p_{i}} S_{1}=\varepsilon_{p_{i}} T M=M_{p_{i}} \cong K_{2 p_{i}} \oplus \cdots \oplus K_{m p_{i}}
$$

where each of the modules $K_{j p_{i}}$ is isomorphic either to $Z\left(p_{i}^{k_{j}}\right)$ or to $\widehat{Z}_{p_{i}}$. Therefore,

$$
S_{1} \cong \bigoplus_{i=1}^{t} K_{2 p_{i}} \oplus \cdots \oplus \bigoplus_{i=1}^{t} K_{m p_{i}} \cong K_{\chi_{2}} \oplus \cdots \oplus K_{\chi_{m}} .
$$

Thus, $M \cong R_{\chi_{1}} \oplus K_{\chi_{2}} \oplus \cdots \oplus K_{\chi_{m}}$. 
Observe that the $R$-module $S_{1}$ occurring in the proof of Theorem 3.1 has pseudorational rank 0 , and there are no other restrictions imposed on that module. Thus, we may assume that $S_{1}$ is an arbitrary finitely generated $R$-module of pseudorational rank 0 . Taking into account the decomposition of $S_{1}$ obtained in the above proof, we arrive at the following statement.

Corollary 3.1. If $M$ is a finitely generated $R$-module of pseudorational rank 0 , then

$$
M \cong K_{\chi_{1}} \oplus \cdots \oplus K_{\chi_{m}}
$$

where $\chi_{1}, \ldots, \chi_{m}$ is a sequence of almost zero characteristics.

Corollary 3.2. The generalized characteristics constitute a complete and independent system of invariants for the class of finitely generated $R$-modules whose pseudorational rank is equal to 0 or 1 .

\section{$\S 4$. Decomposability of Certain $R$-modules}

Let $M$ be a finitely generated $R$-module. We denote by $\rho(M)$ the smallest number of generators of the module $M$. It is clear that $\rho(M) \geq r^{*}(M)$.

Theorem 4.1. If the generalized characteristic of a finitely generated reduced $R$-module $M$ consists of equal characteristics $\chi_{1}, \chi_{2}, \ldots, \chi_{n}$, and the number of these characteristics is equal to $\rho(M)$, then

$$
M \cong R_{\chi_{1}} \oplus R_{\chi_{2}} \oplus \cdots \oplus R_{\chi_{n}} \quad \text { or } \quad M \cong K_{\chi_{1}} \oplus K_{\chi_{2}} \oplus \cdots \oplus K_{\chi_{n}}
$$

Proof. Let $M$ be a finitely generated $R$-module, and let the sequence $\left(\chi_{1}, \chi_{2}, \ldots, \chi_{n}\right)$ be its generalized characteristic; assume that

$$
\chi_{1}=\chi_{2}=\cdots=\chi_{n}=\chi \quad \text { and } n=\rho(M) .
$$

We consider two cases.

Case 1. The characteristic $\chi$ contains infinitely many nonzero elements. Let $P_{1}=$ $\left\{p \in P \mid \chi_{p} \neq 0\right\}$. Since $\rho(M)=n$, there exist elements $x_{1}, \ldots, x_{n} \in M$ such that $\left\langle x_{1}, \ldots, x_{n}\right\rangle_{R}=M$. Consider an arbitrary combination

$$
r_{1} x_{1}+\cdots+r_{n} x_{n}=0, \quad \text { where } r_{1}, \ldots, r_{n} \in R .
$$

For each prime integer $p$, (4.1) induces the combination

$$
\alpha_{p 1} \varepsilon_{p} x_{1}+\cdots+\alpha_{p n} \varepsilon_{p} x_{n}=0, \quad \text { where } \alpha_{p 1}=\varepsilon_{p} r_{1}, \ldots, \alpha_{p n}=\varepsilon_{p} r_{n}
$$

Recalling the restrictions imposed on the characteristic, we see that

$$
\left\langle\varepsilon_{p} x_{1}, \ldots, \varepsilon_{p} x_{n}\right\rangle_{\widehat{Z}_{p}}= \begin{cases}0 & \text { if } \chi_{p}=0 \\ \bigoplus_{n} Z\left(p^{m_{p}}\right) & \text { if } 0<\chi_{p}=m_{p}<\infty, \\ \bigoplus_{n} \widehat{Z}_{p} & \text { if } \chi_{p}=\infty\end{cases}
$$

Formulas (4.2) and (4.3) imply that

$$
\chi_{p}=\infty \Longrightarrow \alpha_{p 1}=\cdots=\alpha_{p n}=0 \quad \text { and } \quad \chi_{p}=m_{p} \in \mathbb{N} \Longrightarrow \alpha_{p 1}, \ldots, \alpha_{p n} \in p^{m_{p}} \widehat{Z}_{p} .
$$

If the characteristic $\chi$ contains infinitely many $\infty$ symbols, then infinitely many $p$ components of the coefficients $r_{1}, \ldots, r_{n}$ in (4.1) are equal to 0 , which means, by properties $1^{0}$ and $2^{0}$ of the ring of pseudorational numbers, that these coefficients belong to the ideal $T$.

If the characteristic $\chi$ contains only a finite number of $\infty$ symbols, then it contains infinitely many $p$-components that are positive integers. Consider the set $P_{2}=\{p \in P \mid$ $\left.\chi_{p} \in \mathbb{N}\right\}$. Since a prime integer $p$ divides a pseudorational number $r$ if and only if $p$ divides 
$\varepsilon_{p} r$, it follows that $p$ divides $r_{1}, \ldots, r_{n}$ for all $p \in P_{2}$. But a pseudorational number has infinitely many prime divisors only if it belongs to $T$. Therefore, $r_{1}, \ldots, r_{n} \in T$.

Thus, we have proved that the elements $x_{1}, \ldots, x_{n}$ are independent modulo $T M$, which means that $r^{*}(M)=n$. Now, by Proposition 2.2, we have $r^{*}\left(\Delta M_{X}\right)=0$ and

$$
\Delta M_{X}=\bigoplus_{p \in P} \varepsilon_{p} \Delta M_{X}=\bigoplus_{p \in P} K_{p},
$$

where $K_{p}=\bigoplus_{n} p^{m_{p}} \widehat{Z}_{p}$ if $0 \leq \chi_{p}=m_{p}<\infty$, and $K_{p}=0$ if $\chi_{p}=\infty$.

In the $R$-module $L=R_{\chi_{1}} \oplus R_{\chi_{2}} \oplus \cdots \oplus R_{\chi_{n}}$, we take a system of elements $Y=$ $\left\{y_{1}, \ldots, y_{n}\right\}$, where

$$
y_{1}=(1,0, \ldots, 0), y_{2}=(0,1, \ldots, 0), \ldots, y_{n}=(0,0, \ldots, 1) .
$$

Obviously, $Y$ is a generating system of the module $L$, and $\Delta L_{Y}=\Delta M_{X}$. By Corollary 2.1, the modules $M$ and $L$ are isomorphic.

Case 2. $\chi$ is an almost zero characteristic. Then $r^{*}(M)=0$, and consequently, $M$ is determined by its generalized characteristic, i.e.,

$$
M \cong K_{\chi_{1}} \oplus K_{\chi_{2}} \oplus \cdots \oplus K_{\chi_{n}} .
$$

Corollary 4.1. If the generalized characteristic of a finitely generated reduced $R$-module $M$ consists of characteristics $\chi_{1}, \chi_{2}, \ldots, \chi_{n}$ that differ only at a finite number of positions, and if $n=\rho(M)$, then

$$
M \cong R_{\chi_{1}} \oplus R_{\chi_{2}} \oplus \cdots \oplus R_{\chi_{n}} \quad \text { or } \quad M \cong K_{\chi_{1}} \oplus K_{\chi_{2}} \oplus \cdots \oplus K_{\chi_{n}} .
$$

Proof. If an $R$-module $M$ satisfies the assumptions of the corollary, then in the ring $R$ there exists an idempotent $(1-\varepsilon)$ such that the module $(1-\varepsilon) M$ satisfies the conditions of Theorem 4.1. If $r^{*}(M)=0$, then

$$
M \cong K_{\chi_{1}} \oplus K_{\chi_{2}} \oplus \cdots \oplus K_{\chi_{n}}
$$

and if $r^{*}(M) \neq 0$, then

$$
M=(1-\varepsilon) M \oplus \varepsilon M \cong R_{\chi_{1}} \oplus R_{\chi_{2}} \oplus \cdots \oplus R_{\chi_{n}} .
$$

\section{§5. Quotient Divisible MiXed Groups}

In this section we consider the categories $\mathcal{Q T} \mathcal{F}$ and $\mathcal{Q D}$, the objects of which are, respectively, the torsion free Abelian groups of finite rank and the quotient divisible mixed groups, and the morphisms of which are quasihomomorphisms.

In 3] it was proved that the categories $\mathcal{Q T} \mathcal{F}$ and $\mathcal{Q D}$ are mutually dual. On the other hand, in [4 Fomin constructed a category $\mathcal{F}$ whose objects are finitely generated $R$-modules with a chosen free generating system, and whose morphisms are pairs of quasihomomorphisms; he proved that the categories $\mathcal{F}$ and $\mathcal{Q D}$ are equivalent. In what follows, we shall try to use the "nearness" of the groups in $\mathcal{Q T} \mathcal{F}$ and $\mathcal{Q D}$ to $R$-modules, in order to extend the above results to these categories.

Definition 5.1 (3]). A group $G$ is said to be quotient divisible if it contains no periodic divisible subgroup but contains a free subgroup $F$ of finite rank such that $G / F$ is a periodic divisible group.

The group $F$ occurring in Definition 5.1 is called the fundamental subgroup, and a fixed linearly independent generating system $X=\left\{x_{1}, \ldots, x_{n}\right\}$ of the group $F$ is the fundamental system of the quotient divisible group $G$.

Let $G$ be any reduced quotient divisible mixed group; we denote by $\widehat{G}$ its $Z$-adic completion. The canonical homomorphism $\alpha: G \rightarrow \widehat{G}$ is a monomorphism, because 
ker $\alpha=\bigcap_{n \in N} n G=G^{1}=0$. The group $\widehat{G}$ is a $\widehat{Z}$-module, and consequently a module over the ring of pseudorational numbers.

Definition 5.2 (4) . The $R$-module $\mathcal{R}(G)=\operatorname{div} G \oplus\langle\alpha(G)\rangle_{R}$ is called the pseudorational type of the quotient divisible group $G$.

The pseudorational rank $r^{*}(G)$ of a quotient divisible group $G$ is defined as the pseudorational rank of its pseudorational type:

$$
r^{*}(G)=r^{*}(\mathcal{R}(G)) .
$$

Obviously, there exists an embedding $\varphi: G \rightarrow \mathcal{R}(G)$; below we shall always identify the group $G$ with its image $\varphi(G)$.

By definition, the generalized characteristic of a quotient divisible group is the generalized characteristic of its pseudorational type.

Theorem 5.1. A quotient divisible group is an R-module if and only if its generalized characteristic is locally free.

Proof. If $G$ is the additive group of an $R$-module, then, for every prime integer $p$,

$$
G=\left(1-\varepsilon_{p}\right) G \oplus \varepsilon_{p} G=\left(1-\varepsilon_{p}\right) G \oplus \widehat{G}_{p} .
$$

In 4 it was proved that $\mathcal{R}(G)$ is a finitely generated $R$-module; therefore, $\widehat{G}_{p}$ is a finitely generated $\widehat{Z}_{p}$-module. Consequently, it decomposes into a direct sum of finitely many cyclic $\widehat{Z}_{p}$-modules. If $\widehat{G}_{p}$ is not finite, then $G$ contains a direct summand of the form $\widehat{Z}_{p}$, which is impossible, because Definition 5.1 implies that $G$ is a group of finite rank. Thus, for every prime integer $p$ the group $\widehat{G}_{p}$ is finite, which means that the generalized characteristic of $G$ is locally free.

Let $G$ be a quotient divisible group whose generalized characteristic is locally free. To prove the "if" part, it suffices to show that $G=\mathcal{R}(G)$, i.e., that $r g \in G$ for all $r \in R$, $g \in G$.

Property $1^{0}$ of the ring of pseudorational numbers shows that each number $r \in R$ can be represented in the form

$$
r=(1-\varepsilon)|r|+\varepsilon r,
$$

where $|r|=\frac{m}{n} \in Q, \varepsilon=\varepsilon_{p_{1}}+\cdots+\varepsilon_{p_{k}}$ is an idempotent of the ring $R$, and all prime divisors of $n$ are contained in the set $\left\{p_{1}, \ldots, p_{k}\right\}$. Since the generalized characteristic of $G$ is locally free, we obtain

$$
G=(1-\varepsilon) G \oplus \varepsilon G,
$$

where the group $(1-\varepsilon) G$ is $p$-divisible for each $p \in\left\{p_{1}, \ldots, p_{k}\right\}$. If $g \in G$, then

$$
r g=(1-\varepsilon) \frac{m}{n} g+\varepsilon r g .
$$

But we have proved above that the element $(1-\varepsilon) \frac{m}{n} g$ is contained in $(1-\varepsilon) G$, and the element $\varepsilon r g$ is contained in $\varepsilon G$. Thus, by (5.1) and (5.2), we have $r g \in G$, whence $G=\mathcal{R}(G)$.

In 2 it was proved that the class of quotient divisible groups with locally free generalized characteristic coincides with the well-known class $\mathcal{G}$ of self-small groups of finite torsion and free rank, which was introduced by Glaz and Wickless in 6].

The following facts are immediate consequences of Theorem 3.1, Property $9^{0}$, and Theorem 4.1.

Corollary 5.1. If $G$ is a quotient divisible group with locally free generalized characteristic, then $r^{*}(G)=r(G)$. 
Corollary 5.2. Let $G$ be a quotient divisible group with locally free generalized characteristic. $G$ is a group of pseudorational rank 1 if and only if $G \cong R_{\chi}$, where $\chi$ is a characteristic containing no infinite p-component.

Corollary 5.3. If $G$ is a quotient divisible group with locally free generalized characteristic, and if the reduced part of $G$ is a group of pseudorational rank 1 , then

$$
G \cong R_{\chi_{1}} \oplus R_{\chi_{2}} \oplus \cdots \oplus R_{\chi_{n}}
$$

where the characteristics $\chi_{1}, \chi_{2}, \ldots, \chi_{n}$ do not contain the $\infty$ symbols, the characteristic $\chi_{1}$ contains infinitely many nonzero elements, and $\chi_{2}, \ldots, \chi_{n}$ are almost zero characteristics.

Let $G$ be a quotient divisible group, and let $F=\bigoplus_{i=1}^{n} Z x_{i}$ be its fundamental subgroup. We define two sets depending on $G$ :

$$
\begin{aligned}
\nabla G_{X} & =\left\{\left(r_{1}, \ldots, r_{n}\right) \in R^{n} \mid r_{1} x_{1}+\cdots+r_{n} x_{n} \in G\right\}, \\
\Delta G_{X} & =\left\{\left(r_{1}, \ldots, r_{n}\right) \in R^{n} \mid r_{1} x_{1}+\cdots+r_{n} x_{n}=0\right\} .
\end{aligned}
$$

Obviously, $\nabla G_{X}$ is a group, $\Delta G_{X}$ is a module over the ring of pseudorational numbers, and $\Delta G_{X} \subset \nabla G_{X}$. We call $\Delta G_{X}$ the module of pseudorational relations of the quotient divisible group $G$.

Lemma 5.1. If $G$ is a quotient divisible mixed group, then

$$
G \cong \nabla G_{X} / \Delta G_{X}
$$

Proof. Let $\varphi: \nabla G_{X} \rightarrow G$ be the mapping defined by the rule

$$
\varphi\left(r_{1}, \ldots, r_{n}\right)=r_{1} x_{1}+\cdots+r_{n} x_{n} .
$$

This is an epimorphism of groups, and its kernel coincides with $\Delta G_{X}$; the homomorphism theorem yields $G \cong \nabla G_{X} / \Delta G_{X}$.

Theorem 5.2 (4). If $H$ is a reduced $R$-module, or $G$ is a divisible $R$-module, then $\operatorname{Hom}_{Z}(G, H)=\operatorname{Hom}_{R}(G, H)$.

Lemma 5.2. Let $G, H$ be quotient divisible groups; assume that either $H$ is a reduced group, or $G$ is a divisible group. Next, let $\bigoplus_{i=1}^{n} Z x_{i}$ be a fundamental subgroup of $G$, and let $\varphi: G \rightarrow H$ be a group homomorphism. If

$$
g=r_{1} x_{1}+\cdots+r_{n} x_{n} \in G, \quad r_{1}, \ldots, r_{n} \in R
$$

then $\varphi(g)=r_{1} \varphi\left(x_{1}\right)+\cdots+r_{n} \varphi\left(x_{n}\right)$.

Proof. We analyze several cases.

Case 1 . The groups $G$ and $H$ are reduced. Let $\widehat{G}$ and $\widehat{H}$ denote the $Z$-adic completions of the groups $G$ and $H$; there exists a unique homomorphism $\varphi^{*}$ such that the diagram

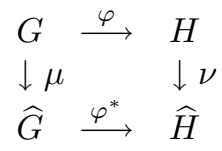

is commutative. Since the mappings $\mu$ and $\nu$ are monomorphisms, we may assume that $G \subset \widehat{G}$ and $H \subset \widehat{H}$. Since $\widehat{G}$ and $\widehat{H}$ are reduced $R$-modules, Theorem 5.2 applies, showing that

$$
\begin{aligned}
\varphi(g) & =\varphi\left(r_{1} x_{1}+\cdots+r_{n} x_{n}\right)=\varphi^{*}\left(r_{1} x_{1}+\cdots+r_{n} x_{n}\right) \\
& =r_{1} \varphi^{*}\left(x_{1}\right)+\cdots+r_{n} \varphi^{*}\left(x_{n}\right)=r_{1} \varphi\left(x_{1}\right)+\cdots+r_{n} \varphi\left(x_{n}\right) .
\end{aligned}
$$


Case 2. The groups $G$ and $H$ are torsion free and divisible. Then they are divisible $R$-modules, and by Theorem 5.2 we have

$$
\varphi(g)=\varphi\left(r_{1} x_{1}+\cdots+r_{n} x_{n}\right)=r_{1} \varphi\left(x_{1}\right)+\cdots+r_{n} \varphi\left(x_{n}\right) .
$$

Case 3. The group $G$ is divisible and $H=D \oplus H_{1}$, where $D$ is a divisible group and $H_{1}$ is a reduced group. Since $\operatorname{Hom}(G, H)=\operatorname{Hom}(G, D)$, this case reduces to Case 2 .

Case 4. The group $H$ is reduced and $G=D \oplus G_{1}$, where $D$ is a divisible group and $G_{1}$ is a reduced group. Since $\operatorname{Hom}(G, H)=\operatorname{Hom}\left(G_{1}, H\right)$, this case reduces to Case 1.

Proposition 5.1. Two quotient divisible mixed groups are isomorphic if and only if they admit equal modules of pseudorational relations.

Proof. Let $G$ and $H$ be quotient divisible mixed groups, and let $\Delta G_{X}$ and $\Delta H_{Y}$ be their modules of pseudorational relations. Suppose

$$
\Delta G_{X}=\Delta H_{Y} .
$$

If $\left(r_{1}, \ldots, r_{n}\right)$ is an element of $\nabla G_{X}$, then

$$
r_{1} x_{1}+\cdots+r_{n} x_{n}=g \in G .
$$

Since $X$ is a fundamental system of $G$, the group $G /\langle X\rangle$ is periodic. Therefore, there exists $m \in \mathbb{N}$ such that

$$
m g=m_{1} x_{1}+\cdots+m_{n} x_{n},
$$

where $m_{1}, \ldots, m_{n}$ are integers. By (5.4) and (5.5), we have

$$
\left(m r_{1}-m_{1}\right) x_{1}+\cdots+\left(m r_{n}-m_{n}\right) x_{n}=0
$$

i.e., $\left(\left(m r_{1}-m_{1}\right), \ldots,\left(m r_{n}-m_{n}\right)\right) \in \Delta G_{X}$. Recalling (5.3), we see that

$$
\left(m r_{1}-m_{1}\right) y_{1}+\cdots+\left(m r_{n}-m_{n}\right) y_{n}=0
$$

or

$$
m\left(r_{1} y_{1}+\cdots+r_{n} y_{n}\right)=m_{1} y_{1}+\cdots+m_{n} y_{n} \in\langle Y\rangle \subset H .
$$

Since $H$ is a pure subgroup of the group $\mathcal{R}(H)$, relation (5.6) implies the existence of an element $h \in H$ such that

$$
m h=m_{1} y_{1}+\cdots+m_{n} y_{n} .
$$

By (5.6) and (5.7), we have $m\left(r_{1} y_{1}+\cdots+r_{n} y_{n}-h\right)=0$, i.e.,

$$
r_{1} y_{1}+\cdots+r_{n} y_{n}-h=t \in t(\mathcal{R}(H)) .
$$

But $t(\mathcal{R}(H))=t(H)$, whence $r_{1} y_{1}+\cdots+r_{n} y_{n}=h-t \in H$, and

$$
\left(r_{1}, \ldots, r_{n}\right) \in \nabla H_{Y} \text {. }
$$

This shows that $\nabla G_{X} \subseteq \nabla H_{Y}$. Similarly we can prove that $\nabla H_{Y} \subseteq \nabla G_{X}$, i.e., $\nabla G_{X}=$ $\nabla H_{Y}$. Thus,

$$
G \cong \nabla G_{X} / \Delta G_{X}=\nabla H_{Y} / \Delta H_{Y} \cong H .
$$

The "only if" part is proved.

Conversely, let $\varphi: G \rightarrow H$ be an isomorphism, and let $X=\left\{x_{1}, \ldots, x_{n}\right\}$ be a fundamental system in $G$. Then $Y=\left\{\varphi\left(x_{1}\right), \ldots, \varphi\left(x_{n}\right)\right\}$ is a fundamental system in $H$; moreover,

$$
r_{1} x_{1}+\cdots+r_{n} x_{n}=0 \Longleftrightarrow r_{1} \varphi\left(x_{1}\right)+\cdots+r_{1} \varphi\left(x_{n}\right)=0,
$$

which means that $\Delta G_{X}=\Delta H_{Y}$. 
Theorem 5.3. If the generalized characteristic of a quotient divisible group $G$ consists of several copies of one and the same characteristic $\chi$, and their number is equal to $r(G)$, then $G \cong \bigoplus_{r(G)} Q_{\chi}$, where $Q_{\chi}$ is a quotient divisible group of rank 1 and characteristic $\chi$.

Proof. Let $G$ be a group satisfying the assumptions of the theorem, and let $X=$ $\left\{x_{1}, \ldots, x_{n}\right\}$ be a fundamental system of this group. We consider two cases.

Case 1. $G$ is a reduced group.

In 44 it was shown that $X$ is a generating system of the pseudorational type of the group $G$, i.e., $\mathcal{R}(G)=\left\langle x_{1}, \ldots, x_{n}\right\rangle_{R}$. Precisely as in the proof of Theorem 4.1, it can be shown that the system $X$ is independent modulo $T \mathcal{R}(G)$. Therefore, $r(G)=r^{*}(\mathcal{R}(G))=$ $n$. It follows that $X$ is a minimal generating system of the module $\mathcal{R}(G)$, which means that $\mathcal{R}(G)$ satisfies the assumptions of Theorem 4.1. Hence,

$$
\mathcal{R}(G) \cong \bigoplus_{n} R_{\chi}
$$

This implies that $r^{*}(\mathcal{R}(G))=n$; hence, by Proposition $2.2, r^{*}\left(\Delta \mathcal{R}(G)_{X}\right)=r^{*}\left(\Delta G_{X}\right)=$ 0 . Therefore,

$$
\Delta G_{X}=\bigoplus_{p \in P} \varepsilon_{p} \Delta G_{X}=\bigoplus_{p \in P} K_{p},
$$

where $K_{p}=\bigoplus_{n} p^{m_{p}} \widehat{Z}_{p}$ if $0 \leq \chi_{p}=m_{p}<\infty$, and $K_{p}=0$ if $\chi_{p}=\infty$.

Let $H=\bigoplus_{n} Q_{\chi}$. Since the decomposition (5.10) of the module $\Delta G_{X}$ is completely determined by the generalized characteristic of the group $G$, and the group $H$ has the same generalized characteristic, we obtain $\Delta G=\Delta H$. Thus, the groups $G$ and $H$ are isomorphic by Proposition 5.1.

Case 2. $G=\operatorname{div} G \oplus G / \operatorname{div} G$ and $\operatorname{div} G \neq 0$.

Observe that the pseudorational types of the groups $G$ and $G / \operatorname{div} G$ differ only in the direct summand $\operatorname{div} G$; consequently, their generalized characteristics coincide. Let

$$
z_{1}=x_{1}+\operatorname{div} G, z_{2}=x_{2}+\operatorname{div} G, \ldots, z_{n}=x_{n}+\operatorname{div} G .
$$

Then $\mathcal{R}(G / \operatorname{div} G)=\left\langle z_{1}, \ldots, z_{n}\right\rangle_{R}$. The conditions of the theorem imply that

$$
\left\langle z_{1}\right\rangle_{\widehat{Z}_{p}} \cong\left\langle z_{2}\right\rangle_{\widehat{Z}_{p}} \cong \cdots \cong\left\langle z_{n}\right\rangle_{\widehat{Z}_{p}}
$$

for every prime integer $p$. Thus $o\left(\varepsilon_{p} z_{1}\right)=\cdots=o\left(\varepsilon_{p} z_{n}\right)$ for each prime $p$, which means that $o\left(z_{1}\right)=\cdots=o\left(z_{n}\right)$. Since the system $\left\{x_{1}, \ldots, x_{n}\right\}$ is independent, we see that either $r(G / \operatorname{div} G)=r(G)$ or $r(G / \operatorname{div} G)=0$. Since $\operatorname{div} G \neq 0$, we conclude that $r(G / \operatorname{div} G)=0$, i.e., $r(\operatorname{div} G)=r(G)$. Consequently, the group $G / \operatorname{div} G$ is periodic.

Thus, $G / \operatorname{div} G$ is a quotient divisible group, the generalized characteristic of which is locally free and has pseudorational rank 0. Now, by Theorem 5.1 and Corollary 3.1, we have $G \cong \bigoplus_{n} Q_{\chi}$, where $Q_{\chi}=Q \oplus K_{\chi}$ is a quotient divisible group of rank 1 .

Observe that a quotient divisible group $Q_{\chi}$ of rank 1 is determined (up to isomorphism) by its characteristic $\chi$ and can be described as the pure hull of 1 in the group $R_{\chi}$. In particular, if $\chi$ does not contain the $\infty$ symbols, then $Q_{\chi}=R_{\chi}$.

Corollary 5.4. If the generalized characteristic of a quotient divisible mixed group consists of characteristics of one and the same type, and the number of these characteristics is equal to the rank of the group, then this group decomposes into a direct sum of quotient divisible groups of rank 1.

${ }^{2}$ Recall that $o(x)$ is the standard notation for the order of the element $x$; see 1 . 
Proof. Let $G$ be a group satisfying the assumptions of the corollary, and let $\left(\chi_{1}, \ldots, \chi_{n}\right)$ be its generalized characteristic:

$$
\chi_{1}=\left(m_{1 p}\right)_{p \in P}, \chi_{2}=\left(m_{2 p}\right)_{p \in P}, \ldots, \chi_{n}=\left(m_{n p}\right)_{p \in P} .
$$

Consider the set $P_{1}=\left\{p_{1}, \ldots, p_{k}\right\}$ of all prime integers such that at least one of the equalities

$$
m_{1 p}=m_{2 p}=\cdots=m_{n p}
$$

fails. Since all $m_{i p_{j}}(1 \leq i \leq n, 1 \leq j \leq k)$ are nonnegative integers, the groups $\varepsilon_{p} \mathcal{R}(G)=\widehat{G}_{p}=G_{p}$ are finite for all $p \in P_{1}$. Let $\varepsilon=\varepsilon_{p_{1}}+\cdots+\varepsilon_{p_{k}}$; we have the direct decomposition $G=(1-\varepsilon) G \oplus \varepsilon G$. Obviously, $(1-\varepsilon) G$ is a quotient divisible group satisfying the requirements of Theorem 5.3, and $\varepsilon G$ is an $R$-module of pseudorational rank 0 . Hence,

$$
G=(1-\varepsilon) G \oplus \varepsilon G \cong \bigoplus_{n} Q_{\chi} \oplus K_{\varphi_{1}} \oplus \cdots \oplus K_{\varphi_{n}}=Q_{\chi_{1}} \oplus \cdots \oplus Q_{\chi_{n}} .
$$

For torsion free groups of finite rank, there is a result similar to Corollary 5.4. Namely, in [15] it was proved that if the Richman type of a torsion free group of finite rank consists of several copies of one and the same type and the number of these copies is equal to the rank of the group, then the group is completely decomposable; i.e., it decomposes into a direct sum of groups of rank 1 .

\section{REFERENCES}

[1] L. Fuchs, Infinite abelian groups. Vols. I, II, Pure Appl. Math., vols. 36, 36-II, Acad. Press, New York-London, 1970, 1973. MR0255673 (41:333) MR0349869 (50:2362)

[2] A. A. Fomin, Some mixed abelian groups as modules over the ring of pseudo-rational numbers, Abelian Groups and Modules (Dublin, 1988), Trends Math., Birkhäuser Verlag, Basel, 1999, pp. 87100. MR 1735560 (2001b:20096)

[3] A. A. Fomin and W. Wickless, Quotient divisible abelian groups, Proc. Amer. Math. Soc. 126 (1998), 45-52. MR:1443826 (98c:20100)

[4] A. A. Fomin, Quotient divisible mixed groups, Abelian Groups, Rings and Modules (Perth, 2000), Contemp. Math., vol. 273, Amer. Math. Soc., Providence, RI, 2001, pp. 117-128. MR1817156 (2002b:20079)

[5] L. Fuchs and K. M. Rangaswamy, On generalized regular rings, Math. Z. 107 (1968), 71-81. MR0233850 (38:2171)

[6] S. Glaz and W. Wickless, Regular and principal projective endomorphism rings of mixed abelian groups, Comm. Algebra 22 (1994), 1161-1176. MR1261253 (95a:20060)

[7] W. Wickless, A functor from mixed groups to torsion-free groups, Abelian Group Theory and Related Topics (Oberwolfach, 1993), Contemp. Math., vol. 171, Amer. Math. Soc., Providence, RI, 1994, pp. 407-417. MR.1293158 (95k:20090)

[8] U. E. Albrecht, Mixed abelian groups with Artinian quasi-endomorphism ring, Comm. Algebra 25 (1997), 3497-3511. MR 1468818 (98j:20081)

[9] A. A. Fomin and W. Wickless, Self-small mixed abelian groups $G$ with $G / T(G)$ finite rank divisible, Comm. Algebra 26 (1998), 3563-3580. MR 1647118 (99j:20061)

[10] _ Categories of mixed and torsion-free finite rank abelian groups, Abelian Groups and Modules (Padova, 1994), Math. Appl., vol. 343, Kluwer Acad. Publ., Dordrecht, 1995, pp. 185-192. MR 1378197 (97c:20083)

[11] A. V. Yakovlev and N'Famara Kamara, Mixed abelian groups of finite rank and their direct decompositions, Vestnik S.-Peterburg. Univ. Mat. Mekh. Astronom. 1993, vyp. 2, 57-61; English transl., Vestnik St. Petersburg Univ. Math. 26 (1993), no. 2, 50-53. MR1370233 (96k:20112)

[12] P. A. Krylov, Mixed abelian groups as modules over their endomorphism rings, Fundam. Prikl. Mat. 6 (2000), no. 3, 793-812. (Russian) MR1801329 (2001m:20099)

[13] P. A. Krylov, E. G. Pakhomova, and E. I. Podberezina, On a class of mixed abelian groups, Vestnik Tomsk. Univ. 269 (2000). (Russian).

[14] S. V. Cheglyakova, Injective modules over the ring of pseudorational numbers, Fundam. Prikl. Mat. 7 (2001), no. 2, 627-629. (Russian) MR 1866471(2002h:16007) 
[15] D. M. Arnold, Finite rank abelian torsion free abelian groups and rings, Lecture Notes in Math., vol. 931, Springer-Verlag, Berlin-New York, 1982. MR665251 (84d:20002)

[16] A. V. Tsarev, The pseudorational rank of an abelian group, Sibirsk. Mat. Zh. 46 (2005), no. 1, 217-229; English transl., Siberian Math. J. 46 (2005), no. 1, 172-181. MR2141315 (2006a:20099)

Moscow State Pedagogical University, Moscow, Russia

E-mail address: an-tsarev@yandex.ru

Received 21/NOV/2005

Translated by A. V. YAKOVLEV 